\title{
Comparative study and performance analysis of Routing protocols for MANET
}

\author{
Er. Mahdi Abdulkader Salem \\ (Department of computer sciences and IT / SHIATS Institute, India)
}

\begin{abstract}
Wireless communication between mobile users is becoming more popular than ever before. This is due to recent technological advances in laptop computers and wireless data communication devices, such as wireless modems and wireless LANs. This has lead to lower prices and higher data rates, which are the two main reasons why mobile computing continues to enjoy rapid growth. With current advances in technology, wireless networks are increasing in popularity. Wireless networks allow users the freedom to travel from one location to another without interruption of their computing services. However, wireless networks require the existence of a wired base station (BS) in order for the wireless user to send/receive messages. Ad-hoc networks, a subset of wireless networks, allow the formation of a wireless network without the need for a BS. All participating users in an Ad-hoc network agree to accept and forward messages, to and from each other. With this flexibility, wireless networks have the ability to form anywhere, at any time, as long as two or more wireless users are willing to communicate.

This chapter will introduce the mobile ad hoc networking in general, provide background on the nature and problems of this type of networking and give an overview of the current state of research.

Mobile networking is one of the most important technologies supporting pervasive computing. During the last decade, advances in both hardware and software techniques have resulted in mobile hosts and wireless networking common and miscellaneous. Generally there are two distinct approaches for enabling wireless mobile units to communicate with each other.
\end{abstract}

Keywords: Mobile ad hoc networks (MANETS), Quality of services (QoS) , AODV, ZRP, DSDV routing.

\section{Introduction}

Wireless communication between mobile users is becoming more popular than ever before. This is due to recent technological advances in laptop computers and wireless data communication devices, such as wireless modems and wireless LANs. This has lead to lower prices and higher data rates, which are the two main reasons why mobile computing continues to enjoy rapid growth. With current advances in technology, wireless networks are increasing in popularity. Wireless networks allow users the freedom to travel from one location to another without interruption of their computing services. However, wireless networks require the existence of a wired base station (BS) in order for the wireless user to send/receive messages. Ad-hoc networks, a subset of wireless networks, allow the formation of a wireless network without the need for a BS. All participating users in an Ad-hoc network agree to accept and forward messages, to and from each other. With this flexibility, wireless networks have the ability to form anywhere, at any time, as long as two or more wireless users are willing to communicate.

This chapter will introduce the mobile ad hoc networking in general, provide background on the nature and problems of this type of networking and give an overview of the current state of research.

\section{Infrastructure}

Wireless mobile networks have traditionally been based on the cellular concept and relied on good infrastructure support, in which mobile devices communicate with access points like base stations connected to the fixed network infrastructure. Typical examples of this kind of wireless networks are GSM, WLL, WLAN, etc.

\section{Infrastructure-less}

In infrastructure-less approach, the mobile wireless network is commonly known as a Mobile Ad-hoc Network (MANET) [1,2]. A MANET is a collection of wireless nodes that can dynamically form a network to exchange information without using any pre-existing fixed network infrastructure. This is a very important part of communication technology that supports truly pervasive computing, because in many contexts information exchange between mobile units cannot rely on any fixed network infrastructure, but on rapid configuration of a wireless connections on-the-fly. Wireless Ad-hoc networks themselves are an independent, wide area of research and applications, instead of being only just a complement of the cellular system. 


\section{MANET Concept}

A mobile ad hoc network is a collection of wireless nodes that can dynamically be set up anywhere and anytime without using any pre-existing network infrastructure. It is an autonomous system in which mobile hosts connected by wireless links are free to move randomly and often act as routers at the same time. The traffic types in Ad-hoc networks are quite different from those in an infrastructure wireless network, including:

\section{a) Peer-To-Peer}

Communication between two nodes which are within one hop. Network traffic (Bps) is usually consistent.

\section{b) Remote-To-Remote}

Communication between two nodes beyond a single hop but which maintain a stable route between them. This may be the result of several nodes staying within communication range of each other in a single area or possibly moving as a group. The traffic is similar to standard network traffic.

\section{c) Dynamic Traffic}

This occurs when nodes are dynamic and moving around. In this case routes must be reconstructed. This results in a poor connectivity and network activity in short bursts. Figure 1.1 shows the examples of both infrastructure and infrastructureless Ad-hoc wireless networks.
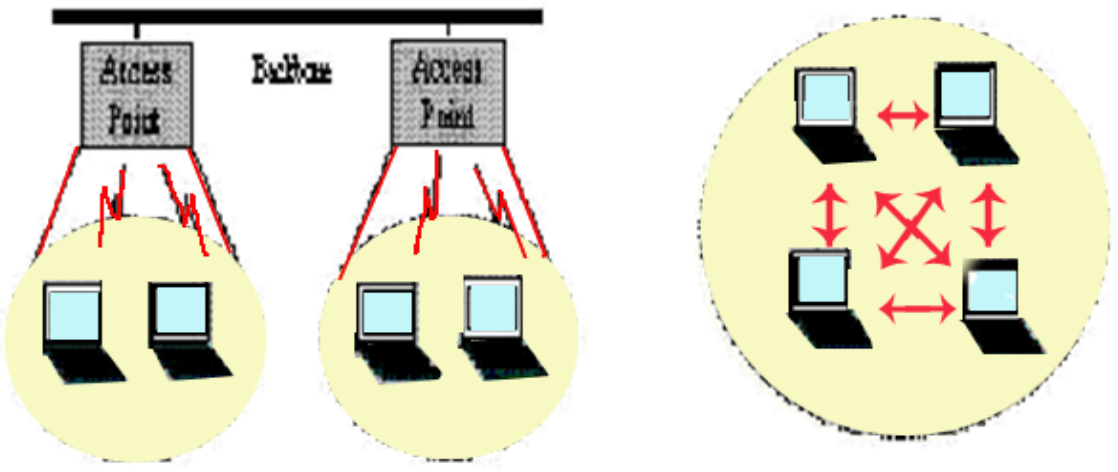

\section{Figure 1.1 Infrastructured and Infrastructure-less Ad-hoc wireless networks}

A "mobile ad hoc network" (MANET) is an autonomous system of mobile routers (and associated hosts) connected by wireless links - the union of which form an arbitrary graph. The routers are free to move randomly and organize themselves arbitrarily; thus, the network's wireless topology may change rapidly and unpredictably. Such a network may operate in a stand-alone fashion, or may be connected to the larger Internet. The current charter of the MANET IETF working group no longer lists a specific definition, reflecting the fact that a wide range of communication forms can be considered a MANET. Mobile ad hoc networks have been the subject of a great deal of recent and challenging research efforts by many excellent scientists and Internet pioneers. These have ranged from very general to very special issues, covering any network layer from the physical media characteristics up to security protocols and service location. Mainly driven by military research in the past, MANETs are about to enter the commercial platform as well, as they contribute to general scientific work. This observation is based on the growing interest in research and the existence of the first commercial techniques on the market, which work in an ad hoc fashion (Bluetooth). Many problems still need to be solved. Establishing and maintaining data connections for various applications between mobile nodes without any given infrastructure or even reliable cooperation, is a complex task that cannot be solved in a general way. The amount of proposed routing protocols and algorithms for MANETs reflects this nature of the problem. 


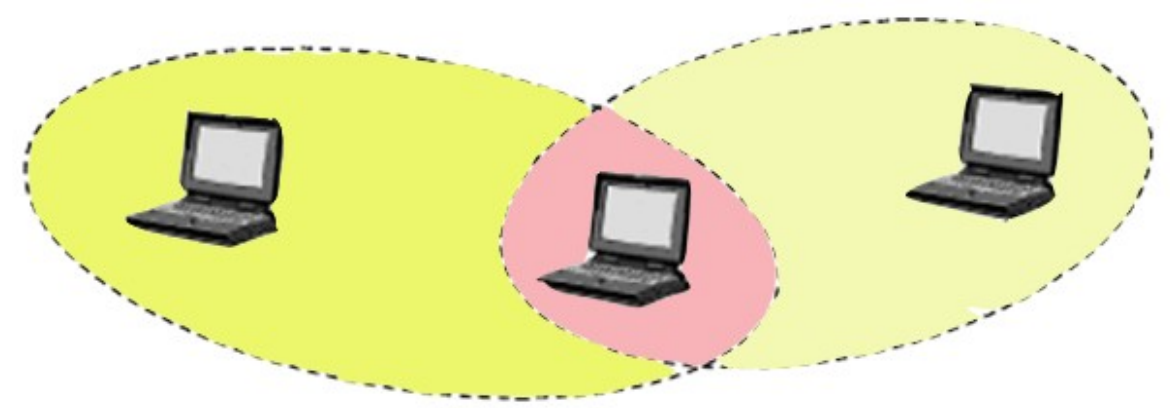

Figure 1.2 Example of Simple Ad-hoc Network with three participating Nodes

Figure 1.2 shows a simple Ad-hoc network with three nodes. The outermost nodes are not within transmitter range of each other. However the middle node can be used to forward packets between the outermost nodes. The middle node is acting as a router and the three nodes have formed an Ad-hoc network.

An Ad-hoc network uses no centralized administration. This is to be sure that the network won't collapse just because one of the mobile nodes moves out of transmitter range of the others. Nodes should be able to enter/leave the network as they wish. Because of the limited transmitter range of the nodes, multiple hops may be needed to reach other nodes. Every node wishing to participate in an Ad-hoc network must be willing to forward packets for other nodes. Thus every node acts both as a host and as a router. A node can be viewed as an abstract entity consisting of a router

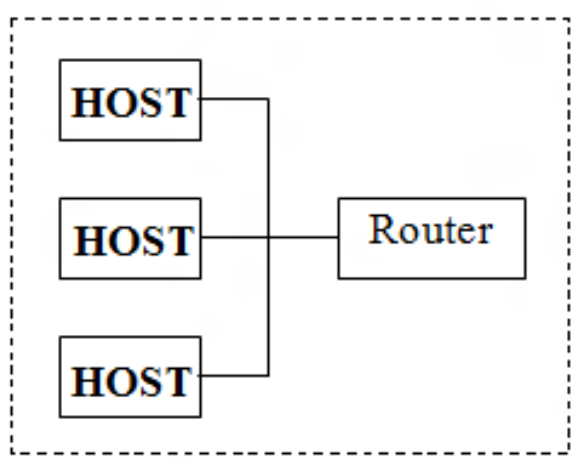

Figure 1.3 Block diagram of mobile node acting both as hosts and as router.

And a set of affiliated mobile hosts (Figure 1.3). A router is an entity, which, among other things runs a routing protocol. A mobile host is simply an IP-addressable host/entity in the traditional sense.

Ad-hoc networks are also capable of handling topology changes and malfunctions in nodes. It is fixed through network reconfiguration. For instance, if a node leaves the network and causes link breakages, affected nodes can easily request new routes and the problem will be solved. This will slightly increase the delay, but the network will still be operational.

Wireless Ad-hoc networks take advantage of the nature of the wireless communication medium. In other words, in a wired network the physical cabling is done a priori restricting the connection topology of the nodes. This restriction is not present in the wireless domain and, provided that two nodes are within transmitter range of each other, an instantaneous link between them may be established.

\section{Specific Features of MANET}

Some of the salient features of MANETs are as follows:

\section{Autonomous Terminal}

In MANET, each mobile terminal is an autonomous node, which may function as both a host and a router. In other words, besides the basic processing ability as a host, the mobile nodes can also perform switching functions as a router. So usually endpoints and switches are indistinguishable in MANET.

\section{Distributed Operation}

Since there is no background network for the central control of the network operations, the control and management of the network is distributed among the terminals. The nodes involved in a MANET should collaborate amongst themselves and each node acts as a relay as needed, to implement functions e.g. Security and routing. 
Multi-hop Routing

Basic types of Ad-hoc routing algorithms can be single-hop and multi-hop, based on different link layer attributes and routing protocols. Single-hop MANET is simpler than multi-hop in terms of structure and implementation, with the cost of lesser functionality and applicability. When delivering data packets from a source to its destination out of the direct wireless transmission range, the packets should be forwarded via one or more intermediate nodes.

\section{Dynamic Network Topology}

Since the nodes are mobile, the network topology may change rapidly and unpredictably and the connectivity among the terminals may vary with time. MANET should adapt to the traffic and propagation conditions as well as the mobility patterns of the mobile network nodes. The mobile nodes in the network dynamically establish routing among themselves as they move about, forming their own network on the fly. Moreover, a user in the MANET may not only operate within the Ad-hoc network, but may require access to a public fixed network (e.g. Internet).

\section{Fluctuating Link Capacity}

The nature of high bit-error rates of wireless connection might be more profound in a MANET. One end-to-end path can be shared by several sessions. The channel over which the terminals communicate is subject to noise, fading, and interference, and has less bandwidth than a wired network. In some scenarios, the path between any pair of users can traverse multiple wireless links and the link themselves can be heterogeneous.

\section{Light-Weight Terminals}

In most cases, the MANET nodes are mobile devices with less CPU processing capability, small memory size, and low power storage. Such devices need optimized algorithms and mechanisms that implement the computing and communicating functions.

\section{Challenges to MANETs}

Multi-hop routing in such an environment is a much more complex task than routing in conventional (static) networks. This requires that all characteristics of the task will be considered. These are determined by the characteristics of the media, the behavior of nodes in terms of movement (mobility patterns) and in terms of communication (data and traffic patterns).

- Cooperation between nodes is strongly desired and may need to be encouraged.

- The used transmission medium results in comparatively low bandwidth and a high potential of channel contention.

- Due to the mobility, the links between the nodes are dynamic and can be short-living.

- The set of nodes is not fixed, since nodes may leave and join the network.

- Traffic requirements may be diverse and quickly changing.

\section{Additional Issues in MANETs}

In Ad-hoc Networks some additional networking issues includes the following

Regardless of the attractive applications, the features of MANET introduce several challenges that must be studied carefully before a wide commercial deployment can be expected. These include:

- Infrastructure less

- No fixed routers

- Highly mobile

- Changing topology

- Link instability

- Resource poor

- Limited energy resources

- Limited wireless transmission range

- Broadcast nature of the wireless medium

- Hidden terminal problem

- Packet losses due to transmission errors

- Mobility-induced route changes

- Mobility-induced packet losses

- Battery constraints

- Potentially frequent network partitions 
- $\quad$ Ease of snooping on wireless transmissions (security hazard)

A few pertaining problems to MANETs are:

\section{Routing}

Since the topology of the network is constantly changing, the issue of routing packets between any pair of nodes becomes a challenging task. Most protocols should be based on reactive routing instead of proactive. Multicast routing is another challenge because the multicast tree is no longer static due to the random movement of nodes within the network. Routes between nodes may potentially contain multiple hops, which is more complex than the single hop communication.

\section{Security and Reliability}

In addition to the common vulnerabilities of wireless connection, an Ad-hoc network has its particular security problems due to nasty neighbor relaying packets. The feature of distributed operation requires different schemes of authentication and key management. Further, wireless link characteristics introduce also reliability problems, because of the limited wireless transmission range, the broadcast nature of the wireless medium (e.g. hidden terminal problem), mobility-induced packet losses, and data transmission errors

\section{Quality of Service (QoS)}

Providing different quality of service levels in a constantly changing environment will be a challenge. The inherent stochastic feature of communications quality in a MANET makes it difficult to offer fixed guarantees on the services offered to a device. An adaptive QoS must be Implemented over the traditional resource reservation to support the multimedia services.

\section{MANETs Applications}

With the increase of portable devices as well as progress in wireless communication, ad hoc networking is gaining importance with the increasing number of widespread applications. Ad hoc networking can be applied anywhere where there is little or no communication infrastructure or the existing infrastructure is expensive or inconvenient to use. Ad hoc networking allows the devices to maintain connections to the network as well as easily adding and removing devices to and from the network. The set of applications for MANETs is diverse, ranging from large-scale, mobile, highly dynamic networks, to small, static networks that are constrained by power sources. Besides the legacy applications that move from traditional infrastructure environment into the ad hoc context, a great deal of new services can and will be generated for the new environment. Mobile ad hoc networks can be applied to a large variety of use cases, where conventional networking cannot be applied, because of difficult terrain, lacking cost-effectiveness or other reasons. Examples of such situations are: Military Battlefield, Commercial Sector (for disaster relief efforts), local level (conference or classroom), Personal Area Networks (such as PDA, Cellular phone, laptop) etc.

\subsection{Simulation Environment}

\section{Result \& Discussion}

\subsubsection{Simulation Software}

The network simulator NS-2 [NS-2] is discrete event simulation software for network simulations. Ns2 began as a variant of the REAL network simulator. The NS-2.29 supports simulation for three routing protocols for ad-hoc wireless networks such as AODV, DSDV, and ZRP. Ns-2 is written in C++ programming language and Object Tool Common Language (OTCL).

\subsubsection{Obtained Results}

To run a simulation with NS-2, we wrote the simulation script in OTCL, get the simulation results in an output trace file, and analyze the results by using the AWK command then by using MS-Excel 2010 we plot the graphs. Ns-2 also offers a visual representation of the simulated network by tracing nodes' movements and events and writing them in a network animator (NAM) file.

\subsubsection{Simulation Parameters}

\begin{tabular}{|l|l|}
\hline Simulation Parameter & Value \\
\hline Number of Nodes & 50 \\
\hline Mobility Model & Random Waypoint Model \\
\hline Max Speed & $10 \mathrm{~m} / \mathrm{s}$ \\
\hline Simulation Time & $100 \mathrm{sec}$ \\
\hline Network Area & $500 \mathrm{~m} \mathrm{X} \mathrm{500} \mathrm{m}$ \\
\hline Routing Protocol & AODV, DSDV, ZRP \\
\hline
\end{tabular}

Table 2.1 Simulation Parameters 


\subsection{Discussion}

\subsubsection{Packet Delivery Ratio}

It is the ratio of packets sent by the application that are received by the receivers. Packets successfully delivered to destinations over total number of packets sent. Packet delivery ratio is calculated by dividing the number of packets received by the destination by the number of packets originated by the application layer of the source. It specifies the packet loss rate, which limits the maximum throughput of the network. The better the delivery ratio, the more complete and correct is the routing protocol.

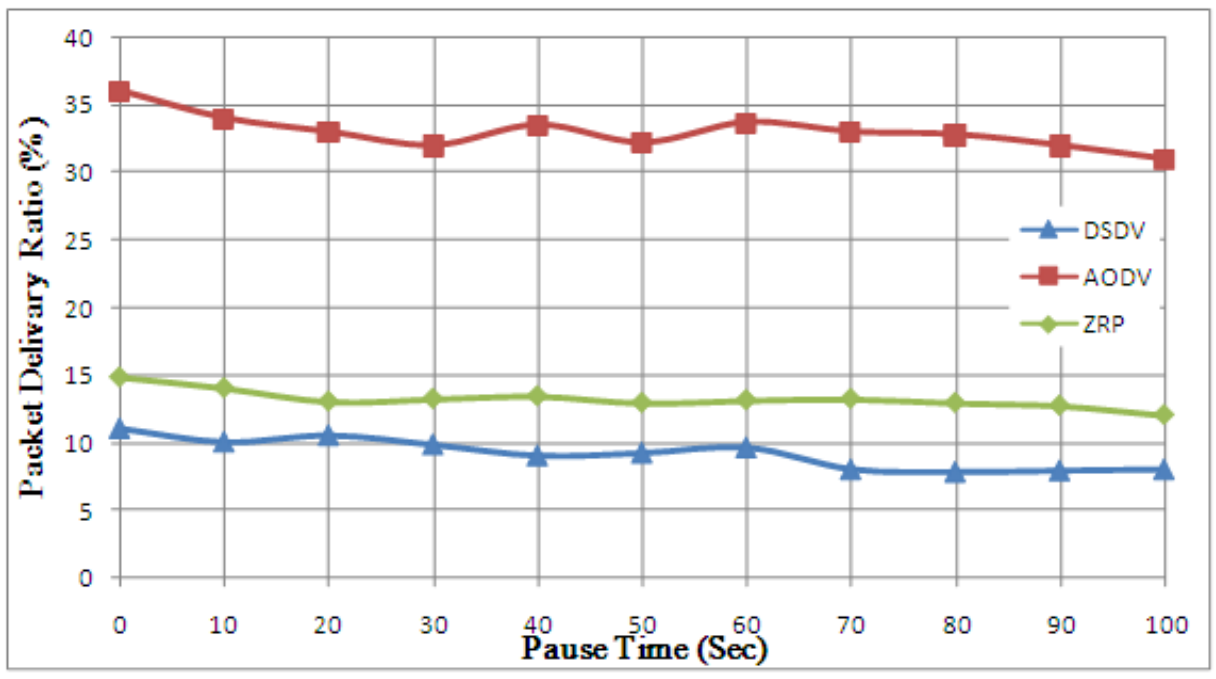

Figure 2.1 Packet Delivery Ratio Performance

Figure 2.1 shows the simulation results of packet delivery ratio verses pause time for 50 nodes. We can notice that AODV has the highest packet delivery fraction. In MANET AODV is purely on-demand routing protocol and in case of the link breakage and route error or route discovery failure AODV sends two times RREQ for getting destination route. Packet delivery fraction of ZRP is better than DSDV. This is because; Zone Routing Protocol has both proactive and reactive nature. DSDV has proactive nature and it cannot form routing table proficiently with the dynamically changing network. During link breakage DSDV fails to resend data. Moreover, it is efficient for cluster and close network nodes. So DSDV has lower performance than other protocols.

\subsubsection{End-To-End Delay}

It indicates how long it took for a packet to travel from the source to the application layer of the destination. It measures the delay a packet suffers after leaving the sender and then arriving at the receiver application. This includes delays due to route discovery, queuing at Internet protocol (IP) and medium access control (MAC) layers, and propagation in the channel.

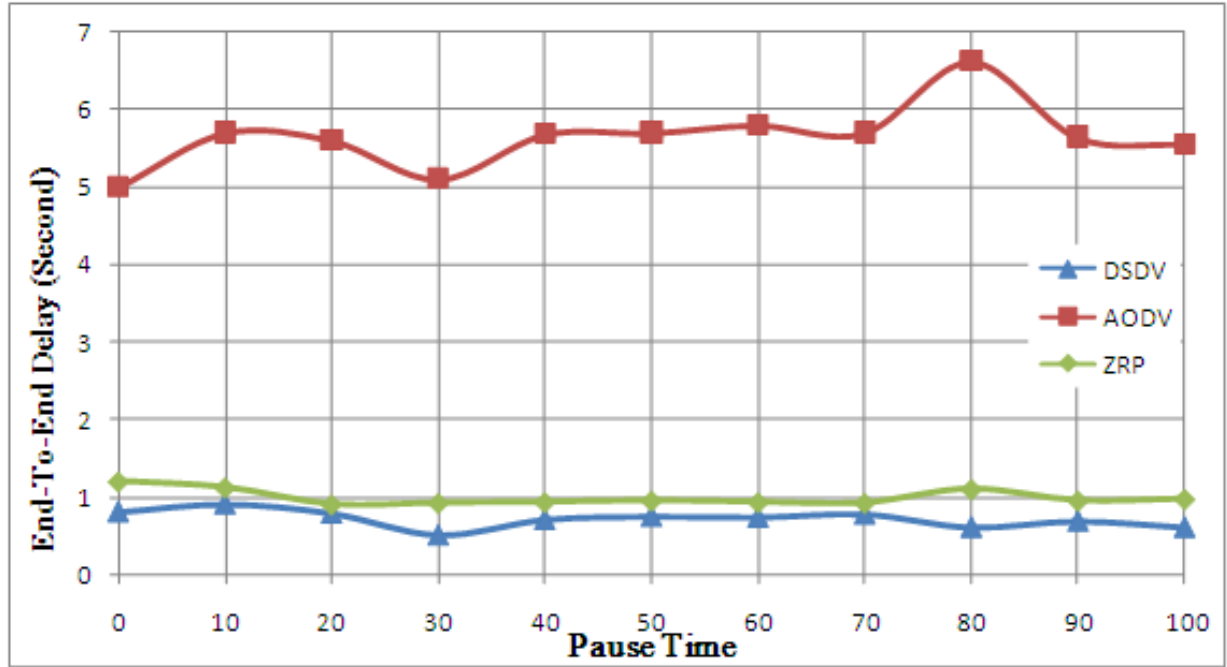

Figure 2.2 End-To-End Delay 
In figure 2.2 shows average end-to-end delay verses pause times are plotted. It shows the average time it took for a packet to travel from the source to destination's application layer. DSDV and ZRP demonstrate lower delay than AODV protocol due to their operation. The presence of routing information in advance leads to lower average end-to-end delay. But AODV shows worst performance in the case of average end-to-end delay. AODV often uses stale routes due to the large route cache, which leads to frequent packet retransmission thus leading to extremely high average end-to-end delay. So as compared to other protocols average end-to-end delay of ZRP and DSDV offers better performance.

\subsubsection{Throughput}

It is defined as the total amount of data a receiver receives from the sender divided by the time it takes for the receiver to get the last packet. Packets delivered per second (TCP traffic only). Examining throughput, especially when it is considered relative to different network scenarios, helps to determine how well the routing protocols permit applications to optimize the use of the available bandwidth.

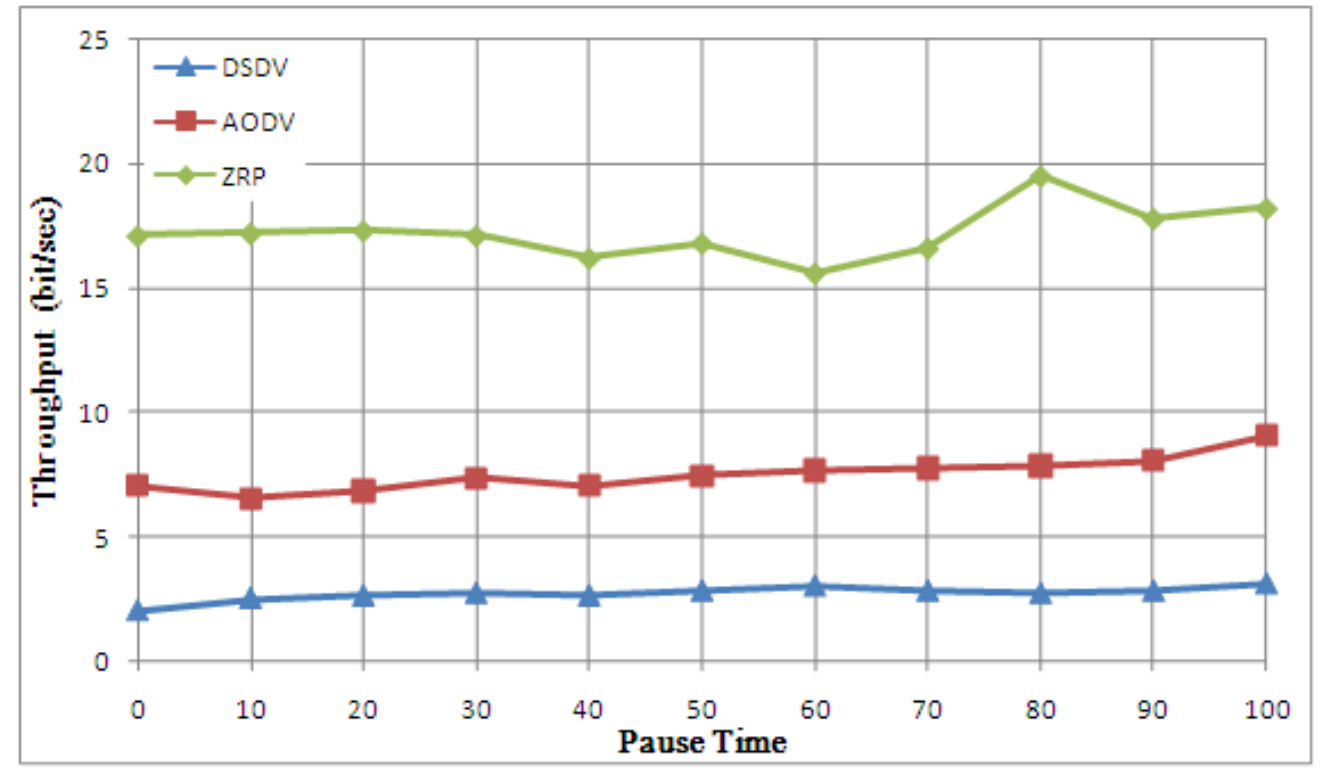

Figure 2.3 Throughput

Figure 2.3 shows the throughput result for 50 source nodes are shown. The graph shows ZRP has highest throughput value than other protocols. ZRP delivers data packets at higher rate because of proactive and reactive characteristics. In ZRP, while sending in INTRA zone routing protocol if it fails to send data or link breakdown occurs then INTER zone routing protocol will be activated. Henceforth data transfer will continue. DSDV has worst performance in throughput than other protocols because most of the nodes cannot participate in data transfer. Another reason is link breakage since DSDV cannot repair route of breakage path. AODV shows good throughput performance than DSDV but less than ZRP.

Table 2.2 Comparison between Ad-hoc routing protocols. of DSDV,AODV and ZRP

\begin{tabular}{|c|c|c|c|c|}
\hline $\begin{array}{l}\text { COMPARISON } \\
\text { PARAME TERS }\end{array}$ & $\begin{array}{l}\text { MANET } \\
\downarrow \text { PROTOCO } \\
\text { LS }\end{array}$ & DSDV & AODV & ZRP \\
\hline Packet Delivery Ratio & & $72 \%$ & $95 \% 100 \%$ & $75.8 \%$ \\
\hline Throughput & & $\min$ & average & Max \\
\hline End to End Delay & & $\min$ & $\max$ & Min \\
\hline
\end{tabular}




\section{Summary}

\section{Summary And Conclusion}

This dissertation describes about MANET and the routing protocols being used in MANET. The main groups of routing protocols have been explained and some of the most commonly used of them were studied. The two main groups of protocols studied are the proactive, hybrid and the reactive protocol. The main characteristic of the proactive is that each node maintains a route to every node in the network. Besides, it periodically updates this information. No matter if there is communication between the nodes or not. As representative example of proactive protocol DSDV was described here. On the other hand, in the reactive ones that is, in AODV the nodes only calculate the routes between those nodes that want to communicate. This kind of protocols performs in a more efficient usage of the bandwidth and the resources of the nodes.

In the reactive, the main problem is the delay to achieve a new route. In the proactive, it is the high usage of resources and bandwidth when it is not necessary. Both, reactive and proactive also have the problem of the scalability. To solve these problems, a new kind of protocols appeared: the hybrid ones. A hybrid routing protocol combines both, the proactive and reactive to achieve better performance. The most popular of them is ZRP and its operation is described here.

\section{Conclusion}

In this dissertation, the performance of AODV, DSDV, and ZRP is compared with respect to three performance metrics, packet delivery ratio, average end-to-end delay and throughput.

AODV shows best performance than DSDV and ZRP in term of packet delivery ratio. DSDV best performs in terms of the average end-to-end delay as compared to AODV and ZRP.

ZRP shows the best performance in terms of throughput when compared to AODV and DSDV.

\section{Future Scope}

In the future work we can evaluate other routing protocols like DYMO for different performance metrics like Network Load and Retransmission attempts.

\section{References}

[1]. David B. Johnson and David A.Maltz, "Dynamic source routing in ad hoc wireless networks". In Mobile Computing, edited by Tomasz Imielinski and Hank Korth, chapter 5, pages 153-181. Kluwer Academic Publishers.

[2]. G. Pei, M. Gerla and T.-W. Chen, (Apr. 2000, pp. D71-D78) Fisheye State Routing in Mobile Ad Hoc Networks. In Proceedings of the 2000 ICDCS Workshops, Taipei,Taiwan,

[3]. Josh Broch et al. (1998), 'A Performance Comparison of Multi Hop Wireless Adhoc Network Routing Protocols', Proceedings of IEEE/ACM Conference on Mobile Computing and Networking, pp. 85-97.

[4]. M. Joa-Ng and I-Tai Lu (1415 1425, 1999) A peer-to-peer zone-based two-level link state routing for mobile ad hoc net-works, IEEE on Selected Areas in Communications, vol. 17, no. 8, pp.

[5]. Neeraj Nehra, R.B. Patel, V.K. Bhat, 2007"Trust Aware Rouitng with Load Balancing in Ad Hoc Network Using Mobile Agent," Advanced Computing and Communications, International Conference on, pp. 454-459, 15th International Conference on Advanced Computing and Communications (ADCOM 2007),. Network Simulator, NS-2, The VINT Project, available from

[6]. Royer E. M. and Toh C. K. (1999), 'A Review of Current Routing Protocols for Ad Hoc Mobile Wireless Networks', IEEE Personal Communications, vol. 6, no. 2, pp. 46-55.

[7]. K. Wu and J. Harms, Oct. 2001"Load sensitive routing for mobile ad hoc networks," Proc. IEEE ICCCN, Phoenix, AZ, pp. 540546 .

[8]. Wang Jianxin et al. (2001), 'QoS routing with Mobility Prediction in MANET', IEEE Conference on Communications, Computers and Signal Processing, (PACRIM’01), vol. 2, pp. 357-360.

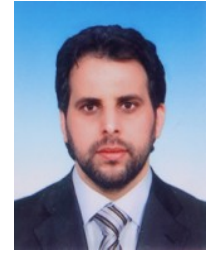

Mahdi Abdulkader Salem has received his B.tech in computer technology in the year 200 from university of Sabha and M.Tech degrees in computer science and information technology from SHIATS Institute Allahabad India in year 2012. He is presently doing Ph.D in computer science and IT from SHIATS Institute India. 